\title{
Traffic Modelling of Low Dense Femtocellular Network for Long Term Evolution
}

\author{
Jesmin Akhter ${ }^{1 *}$, Abu Sayed Md. Mostafizur Rahaman², Md. Imdadul Islam², M. R. Amin ${ }^{3}$ \\ ${ }^{1}$ Institute of Information Technology, Jahangirnagar University, Dhaka, Bangladesh \\ ${ }^{2}$ Department of Computer Science and Engineering, Jahangirnagar University, Dhaka, Bangladesh \\ ${ }^{3}$ Department of Electronics and Communications Engineering, East West University, Dhaka, Bangladesh \\ Email: *jesmin@juniv.edu
}

How to cite this paper: Akhter, J., Rahaman, A.S.Md.M., Islam, Md.I. and Amin, M.R. (2019) Traffic Modelling of Low Dense Femtocellular Network for Long Term Evolution. Journal of Computer and Communications, 7, 88-101.

https://doi.org/10.4236/jcc.2019.712009

Received: November 21, 2019

Accepted: December 20, 2019

Published: December 23, 2019

Copyright (c) 2019 by author(s) and Scientific Research Publishing Inc. This work is licensed under the Creative Commons Attribution International License (CC BY 4.0).

http://creativecommons.org/licenses/by/4.0/

\section{Open Access}

\begin{abstract}
One of the features of Long Term Evolution (LTE) is the deployment of femtocells as the underlain cell of a macrocell without any intervention of frequency planning to offload the traffic. In this paper we used Markov chain to derive the expression of blocking probability for both macro and femtocell in terms of traffic parameters of the network. We developed an analytical model to find the expression of probability of forced termination $(F T)$ using combination of mobility model and probability tree considering low dense femtocellular network. Two different trees were designed: a newly originating call which starts its session in a femtocell and that of in a macro cell. The link parameters of small scale fading of wireless network under Multiple Input Multiple Output (MIMO) are combined with the proposed traffic model to get the probability of $F T$ of a real-life network. A new state transition chain was also developed including its solution for LTE traffic of variable bandwidth (BW) and a comparison was made with Erlang's traffic model.
\end{abstract}

\section{Keywords}

Rayleigh and Nakagami- $m$ Fading, MIMO, RB, Forced Termination, Outage Probability

\section{Introduction}

Major challenge of $4 \mathrm{G} / 5 \mathrm{G}$ mobile cellular network is to enhance the carried traffic capacity. One possible solution is offloading of mobile data traffic from the primary network access to the WiFi infrastructure (for example femtocell of LTE). In [1] and its extended version [2], the authors show the performance of WiFi Direct Device to Device (D2D) technology and demonstrate how cellular 
traffic can be effectively offloaded through a WiFi and provides the estimated gains in energy efficiency and capacity from such offloading. Another big problem of outdoor LTE network is its latency, which directly affects its throughput. In LTE eNB maintains a large queue to reduce overflow of burst traffic at the expense of packet delay. In [3] "Adaptive Receiver-Window Adjustment Algorithm" is used to reduce the latency. Variation of throughput and latency against "modulation and coding scheme" (MCS) and number of users are shown explicitly. Still there is a scope to work on the paper, including other traffic parameters like blocking probability and carried traffic. In [4], instead of conventional traffic of user data, the paper proposed a M2M traffic model to reduce the signaling traffic and bandwidth utilization. In [5] the traffic of LTE-WiMAX integrated network is analyzed including the algorithm of traffic simulation. The variation of FTP, HTTP and voice traffic with time is compared explicitly, where the interference is found as the prime factor. Although femtocell offloads traffic of macro cell but the interference of femto and macro cell can deteriorate throughput of the network. The solution, to limit the interference of femtocells due to their uncontrolled placement is found in [6] [7].

The redundant bits of channel coding degrade the throughput of a network. The compromise between source code rate and channel code rate to minimize the number of redundant bits generated by channel coding with an acceptable Mean Opinion Score (MOS) is analyzed in [8]. Here the authors proposed a dynamic adaptation algorithm of joint source-channel code rate for enhancing voice transmission over LTE network. Paper [9] surveys state-of-art mobile data offloading and proposed to use integrated Femto and Wi-Fi to have improved "service performance" and "interference management" with the constraint of non-overlapping femtocells. The traffic model of low dense femtocell network under fading condition is found in [10] considering the concept of [9] with variable BW.

The rest of the paper is organized as follows: Section 2 provides previous analysis of traffic of LTE network; Section 3 gives the analytical model of Macro-Femto Cellular Network based on Markov chain and probability tree, MIMO channel under fading condition is combined with traffic parameter and traffic model of variable bandwidth (BW). Section 4 provides results based on the analysis of Section 3 and verifies it by simulation and finally, section 5 concludes the entire analysis.

\section{Previous Work}

In [11] the traffic of macro-femto cellular communication system of LTE is modeled by Markov chain. The authors first determined the expression of total call arrival rate of macro and femtocell then separate Markov chains were drawn for macro and femtocell traffic.

The total arrival rate of a macrocell is:

$\lambda_{T m}=\lambda_{o m}+\lambda_{h, m m}+P_{B f} \lambda_{o f}+\alpha P_{D f} \lambda_{h, f f}+(1-\alpha) \lambda_{h, f f}$; where $\lambda_{o m}$ is the newly origi- 
nating call at a macrocell, $\lambda_{h, m m} \rightarrow$ macro to macro handover rate, $P_{B f} \lambda_{o f} \rightarrow$ a call originated in a femtocell and found that all the channels are occupied then the call will use the channel of a macrocell, $\alpha P_{D f} \lambda_{h, f f} \rightarrow$ after a femto to femto handover a UE (user equipment) found that $\operatorname{SINR}>\tau$ (the probability of getting $\operatorname{SINR}>\tau$ is $\alpha$ ) but all the channels of the new femtocell are occupied then the channel of a macrocell will be used, $(1-\alpha) \lambda_{h, f f} \rightarrow$ the case like before but $S I N R<\tau$ hence the channel of a macrocell will be used.

The total arrival rate of a femtocell,

$\lambda_{\text {Tf }}=\left(\lambda_{\text {of }}+\lambda_{h, m f}+\alpha \lambda_{h, f f}+P_{D, m} \beta \lambda_{h, f f}\right) / N$, where $\lambda_{o f} \rightarrow$ the arrival rate of newly originated call in femtocells, $\lambda_{h, m f} \rightarrow$ the rate of handover arrival from macro to femtocells, $\alpha \lambda_{h, f f} \rightarrow$ the femto to femto handover provided that $S I N R>\tau$, where $\tau$ is the threshold $S I N R$ to maintain communication and $P_{D, m} \beta \lambda_{h, f f} \rightarrow$ the femto to femto handover provided that received $S I N R$ lies between two thresholds $\tau_{1}$ and $\tau_{2}$ and $P\left(\tau_{1}<\operatorname{SINR}<\tau_{2}\right)=\beta$.

Traffic offloading strategy of LTE network is analyzed in [12] using two-dimensional Markov chain, where the product of session arrival and session duration is considered offered traffic. Here one dimension is Licensed Assisted LTE Access (LLA) traffic and the other is WiFi AP traffic. The authors consider CTMC with probability state: $\left(N_{W}(t), N_{L}(t)\right)$, where $N_{W}(t)$ is the number of WiFi sessions and $N_{L}(t)$ is the number of offloaded LTE sessions at time $t>0$. The similar traffic model is found in [13], applied for cognitive radio network (CRN), where arrival rate of PU and SU with their termination rate is considered as the offered traffic. Both models were unable to include the variable bandwidth in the state transition chain.

\section{System Model}

\subsection{Traffic Model of Macro-Femto Cellular Network}

In micro-macro cellular system the microcells under a macrocell form the coverage in continuous fashion but the situation is different in LTE with macro-femto combination since femtocells do not form a continuous coverage under a macrocell. Hence the concept of take-back of [14] [15] is not applicable here, visualized from Figure 1 . Let the area of the test macrocell is $A_{m}$ and the

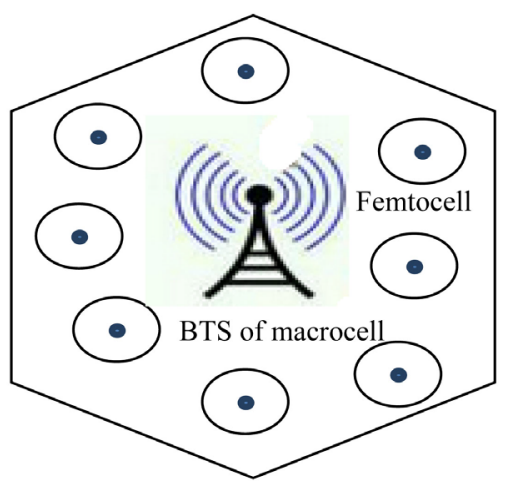

Figure 1. Macro-femto integrated cellular model. 
sum of the area of femtocells within the test macro cell is $A_{f}$ Let the average call arrival rate inside the test cell is $\lambda_{T}$. The arrival rate of macro and femto cells will be distributed as:

Arrival rate of macrocell is $\lambda_{m}=\lambda_{T}\left(1-A_{f} / A_{m}\right)$ and that of femtocells, $\lambda_{f}=\lambda_{T} A_{f} / A_{m}$ In micro-macro cellular system the overflow traffic from microcell is related to macrocell but such concept is not applicable in femto-macro cell hence combined state transition chain of the papers [16] [17] cannot be applied here.

We consider the separates Markov chain of femto and macrocell of [11]; where $n$ is the number of channels (combined data rate/individual user's rate), $N$ is the total number of femtocells within the test macrocell, $\lambda_{f}$ is the call arrival rate within the femtocells and $\lambda_{h}^{\prime}$ is the handover arrival rate from surrounding cells as shown in Figure 2.

The blocking probability under a femtocell is derived as [18]:

$$
B_{f}=\frac{A^{n}\left(\lambda_{f}, \lambda_{h^{\prime}}, N\right) / n !}{\sum_{i=1}^{n} A^{i}\left(\lambda_{f}, \lambda_{h^{\prime}}, N\right) / i !}, \text { where } A\left(\lambda_{f}, \lambda_{h^{\prime}}, N\right)=\frac{\lambda_{f} / N+\lambda_{h^{\prime}}}{\mu_{f}}
$$

Similarly, the call blocking probability under a macrocell is,

$$
B_{m}=\frac{A^{l}\left(\lambda_{m}, \lambda_{h}\right) / l !}{\sum_{i=1}^{l} A^{n}\left(\lambda_{m}, \lambda_{h}\right) / i !}, \text { where } A\left(\lambda_{m}, \lambda_{h}\right)=\left\{\frac{\lambda_{m}+\lambda_{h}}{\mu_{m}}\right\}
$$

and $l$ is the number of channels of the test macrocell, $\lambda_{m}$ is the call arrival rate within the test macrocell and $\lambda_{h}$ is the handover arrival rate from the surrounding cells. The above chain can be improved considering call admission control scheme, where $m$ channels are reserved for handoff calls from the surrounding femtocells. The state transition chain of a macro cell under call admission control is shown in Figure 3.

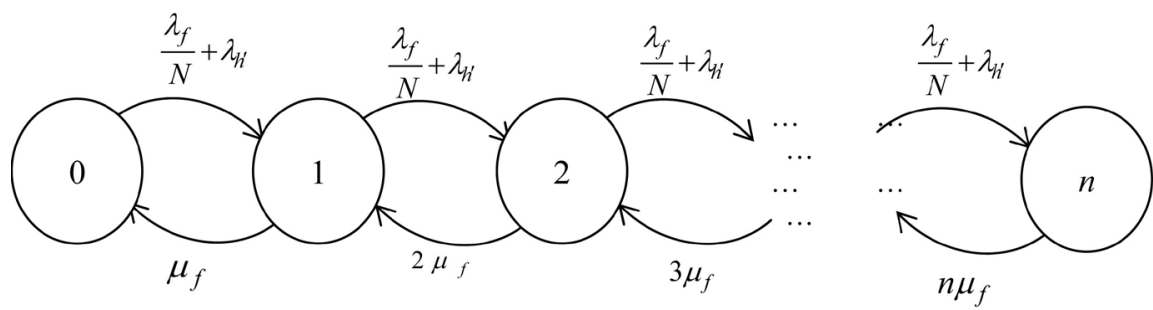

Figure 2. Markov chain of a test femtocell.

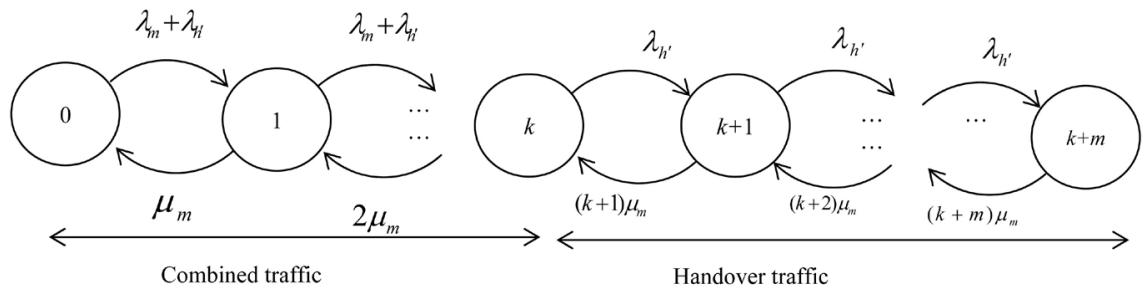

Figure 3. Markov chain of a macrocell under reserved channels for handover calls. 
Applying cut equation like [13] we get in the generalized form as:

$$
p_{x}=\frac{p_{0}\left(A_{m}+A_{h^{\prime}}\right)^{x}}{x !} \text {; where } 0 \leq x \leq k
$$

Similarly,

$$
p_{k+y}=p_{k} \frac{A_{h^{\prime}}^{y}}{\prod_{i=1}^{y}(k+i)} ; \text { where } 1 \leq y \leq m
$$

The probability of $F T$ is,

$$
p_{k+m}=p_{k} \frac{A_{h^{\prime}}^{m}}{\prod_{i=1}^{m}(k+i)}=p_{0} \frac{\left(A_{m}+A_{h^{\prime}}\right)^{k}}{k ! \prod_{i=1}^{m}(k+i)} .
$$

The entire sample space satisfies, $\sum_{i=0}^{k} p_{i}+\sum_{j=1}^{m} p_{k+j}=1$

Applying normalization condition,

$$
p_{0} \sum_{i=0}^{k} \frac{\left(A_{m}+A_{h^{\prime}}\right)^{i}}{i !}+p_{0} \sum_{j=1}^{m} \frac{\left(A_{m}+A_{h^{\prime}}\right)^{k}}{k ! \prod_{l=1}^{j}(k+l)}=1,
$$

we obtain,

$$
p_{0}=\left\{\sum_{i=0}^{k} \frac{\left(A_{m}+A_{h^{\prime}}\right)^{i}}{i !}+\frac{\left(A_{m}+A_{h^{\prime}}\right)^{k}}{k !} \sum_{j=1}^{m} \frac{1}{\prod_{l=1}^{j}(k+l)}\right\}^{-1}
$$

Probability of dropping of newly originating call is $P_{k}$ and probability of forced termination (FT) is $P_{k+m}$. Because of use of low dense femtocells, we assume that two micro and two femtocells are enough to complete a session. In this context we have to determine the probability that an MS crosses the cell border of a femtocell before completing its session as [19] [20],

$$
P_{h f}=\frac{\eta_{f}}{\mu_{f}+\eta_{f}} ;
$$

where $\eta_{f}=2 v / \pi R_{f}, v$ is the velocity of MS, $R_{f}$ is the radius of femtocell and $\mu_{f}$ is the call terminator rate at femtocell. Similarly, for macrocell, the corresponding probability is expressed as,

$$
P_{h m}=\frac{\eta_{m}}{\mu_{m}+\eta_{m}}
$$

We design a probability tree of $F T$ for a MS, $i$ starts its session in a femtocell $i$ as shown in Figure 4 to relate traffic parameters of (1) to (8). The probability of $F T, P_{F T f}$ is derived as,

$$
\begin{aligned}
P_{F T f}= & P_{h m} \cdot B_{m}+P_{h m}\left(1-B_{m}\right) P_{F T m}+P_{h m}\left(1-B_{m}\right) \cdot P_{h f}\left(1-B_{f}\right) \cdot P_{F T f} \\
& +P_{h m}\left(1-B_{m}\right) \cdot P_{h f} B_{f}
\end{aligned}
$$




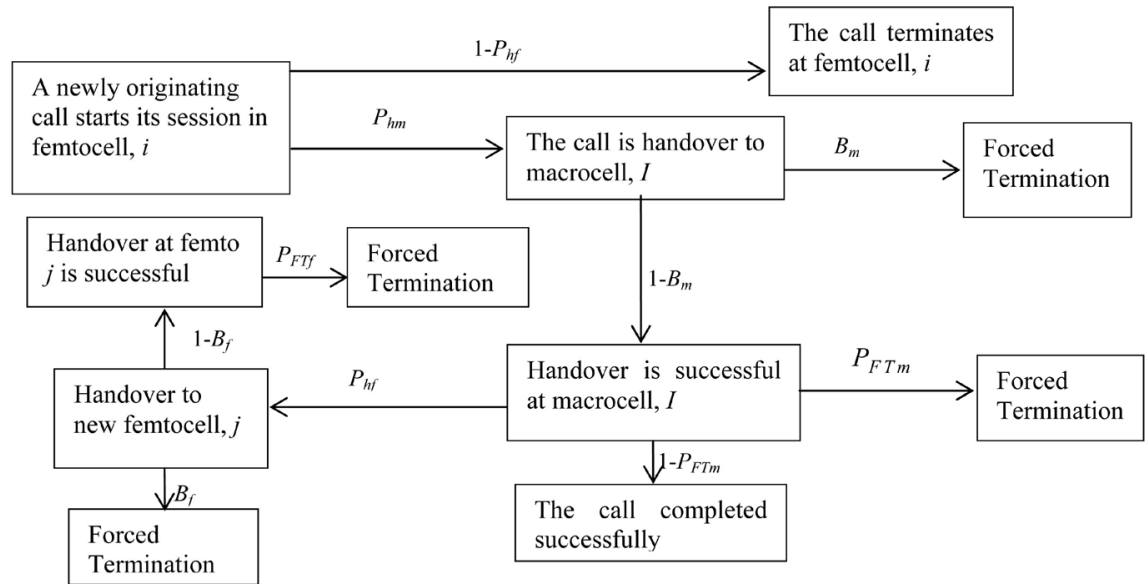

Figure 4. Probability tree of $P_{F T F}$

Another probability tree is designed like Figure 5, when a MS, $j$ starts its session in macro cell $I$ and the corresponding probability of $F T$ is found as:

$$
P_{F T m}=P_{h f}\left(1-B_{f}\right) P_{F T f}+P_{h m} B_{m}+P_{h m}\left(1-B_{m}\right) P_{F T m}+P_{h f} B_{f}
$$

Solving the linear Equation (9) and Equation (10) we can easily obtain $P_{F T m}$ and $P_{F T F}$ Now we have to relate the traffic parameters of this section with small scale fading to get realistic scenario of traffic of wireless network. To combat the fading affect we consider MIMO link discussed in next subsection.

\subsection{The MIMO Channel Model of LTE}

For successful communication both availability of channel of a cell and instantaneous $S N R \geq \tau$ should be ensured; where $\tau$ is the threshold value of instantaneous SNR.

The minimum SNR to maintain QoS of the MIMO link is expressed as [21] [22]:

$$
\gamma_{\min }=\tau=\frac{N_{t} \ln (2)}{E\left\{\operatorname{trace}\left(\boldsymbol{H}^{H} \boldsymbol{H}\right)\right\}}
$$

where $\boldsymbol{H}$ is the $N_{t} \times N_{r}$ MIMO channel matrix, $N_{t}$ is the number of antenna elements of transmit antenna array, $N_{r}$ is that of the receiver antenna array and trace $\left(\boldsymbol{H}^{H} \boldsymbol{H}\right)$ is the sum of diagonal components of the matrix $\boldsymbol{H}^{H} \boldsymbol{H}$.

The trace of $\boldsymbol{H H}^{H}$ can be expressed as,

$$
E\left\{\operatorname{trace}\left(\boldsymbol{H}^{H} \boldsymbol{H}\right)\right\}=N_{t} N_{r} E\left[r^{2}\right]
$$

where $r$ is the random variable of channel gain between the antenna elements. The detail derivation of Equation (11) and Equation (12) are given in [22].

From Equation (11) and Equation (12) we have, $\tau=\frac{\ln (2)}{N_{r} E\left[r^{2}\right]}$ for STBC.

Let us now concentrate on instantaneous and average SNR $\gamma$ and $\gamma_{a v}$ of the MIMO link. First of all, we analyze the MIMO link under TAS (Transmit Antenna 


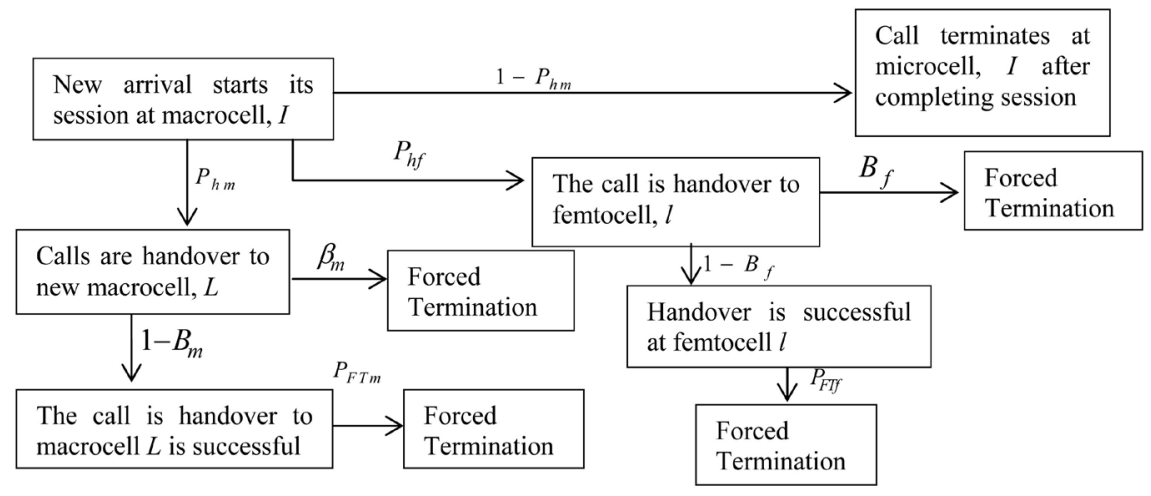

Figure 5. Probability tree of $P_{F T m}$.

Selection) technique then for the complete MIMO. The channel gain vector, selecting the $k$ th antenna element at the transmitting end is,

$$
\boldsymbol{h}_{T R, k}=\left[\begin{array}{lllll}
h_{1, k} & h_{2, k} & h_{3, k} & \ldots & h_{N_{r}, k}
\end{array}\right]
$$

Frobenious norm of $\boldsymbol{h}_{T R, k}$ is,

$$
\left\|\boldsymbol{h}_{T R, k}\right\|=\sqrt{h_{1, k}^{2}+h_{2, k}^{2}+h_{3, k}^{2}+\cdots+h_{N_{r}, k}^{2}}
$$

The transmit antenna element $k \in\left\{1,2,3, \cdots, N_{t}\right\}$ is selected for maximum value of $\left\|\boldsymbol{h}_{T R, k}\right\|$;

The received signal vector is,

$$
\boldsymbol{Y}=\sqrt{P} \boldsymbol{h}_{T R, k}+\boldsymbol{n} ;
$$

where $n$ is the noise vector at the receiving end and $P$ is the transmitted signal power.

The selection combiner at the receiving we choose,

$$
\boldsymbol{h}_{T R, k, s}=\max \left(h_{1, k}, h_{2, k}, h_{3, k}, \cdots, h_{N_{r}, k}\right)
$$

when MRC is used then the weighting vector [23],

$$
\boldsymbol{w}=\frac{\left[\begin{array}{lllll}
h_{1, k}^{*} & h_{2, k}^{*} & h_{3, k}^{*} & \cdots & h_{N_{r}, k}^{*}
\end{array}\right]^{\mathrm{T}}}{\sqrt{h_{1, k}^{2}+h_{2, k}^{2}+h_{3, k}^{2}+\cdots+h_{N_{r}, k}^{2}}}=\frac{\boldsymbol{h}_{T R, k}^{H}}{\left\|\boldsymbol{h}_{T R, k}\right\|}
$$

The output of MRC at the receiver,

$$
y=\sqrt{P}\left\|\boldsymbol{h}_{T R, k}\right\| x+\boldsymbol{w}^{\mathrm{T}} \boldsymbol{n}
$$

The instantaneous SNR of receiver is,

$$
\gamma=\gamma_{a v}\left\|\boldsymbol{h}_{T R, k}\right\|^{2}
$$

If $n_{b}$ is the variance of the noise vector $\boldsymbol{n}$ then,

$$
\gamma_{a v}=\frac{P}{n_{b}}
$$

The weighting vector under TAS of Equation (17) is the contribution of the kth transmit antenna element, hence under the complete MIMO the Equation (17) will be modified as, 


$$
\boldsymbol{w}=\sum_{k=1}^{N_{t}} \frac{\boldsymbol{h}_{T R, k}^{H}}{\left\|\boldsymbol{h}_{T R, k}\right\|}
$$

The pdf of the random variable, $\gamma$ under MRC of the MIMO for Rayleigh fading is like [24],

$$
f_{\Gamma}(\gamma)=\frac{\gamma^{N_{r}-1} \mathrm{e}^{-\frac{\gamma}{\gamma_{a v}}}}{\gamma_{a v}^{N_{r}} \Gamma\left(N_{r}\right)}
$$

that of the Nakagami- $m$ case of $[25]$ is,

$$
f_{\Gamma}(\gamma)=\left(\frac{m}{\gamma_{a v}}\right)^{N_{r}} \frac{\gamma^{m N_{r}-1} \mathrm{e}^{-\frac{m \gamma}{\gamma_{a v}}}}{\Gamma\left(m N_{r}\right)}
$$

and the outage probability,

$$
P_{\text {outage }}(\tau)=\int_{0}^{\tau} f_{\Gamma}(\gamma) \mathrm{d} \gamma
$$

where $\tau$ is the threshold SNR and it depends on the number of received antennas and the variance of the channel gain of the antenna elements.

Now the probability of $F T$, considering the outage probability will be,

$$
P_{\text {FTm_o }}=1-\left(1-P_{F T m}\right)\left(1-P_{\text {out }}(\gamma)\right)
$$

And

$$
P_{\text {FTf_o }}=1-\left(1-P_{F T f}\right)\left(1-P_{\text {out }}(\gamma)\right)
$$

The Equation (25) and Equation (26) provides the probability of $F T$, which depends on the instantaneous SNR, the number of received antenna elements and all traffic parameters will be shown in the result section. In LTE, users deal with traffic of variable BW, hence we have to modify the Markov chain of subsection 3.1. In next subsection we proposed a new state transition chain of variable BW.

\subsection{Traffic of Variable Bandwidth}

In LTE users can take bandwidth on demand. Here we assume the minimum unit of BW of a user is a pair of RB (resource block). In LTE each frame of $10 \mathrm{~ms}$ is divided into 10 subframe of $1 \mathrm{~ms}$, also each sub-frame is divided into two time slots of $0.5 \mathrm{~ms}$ each. Each slot contains either six or seven OFDM symbols, depending on the type of channel coding schemes. When normal Cyclic Prefix (CP) is used then a RB contains 7 symbols under each subcarrier of OFDM, under extended CP, 6 symbols under each sub-carrier. Each RB contains 12 subcarriers, hence $12 \times 6=72$ symbols in a RB of the extended $\mathrm{CP}$ and $12 \times 7=84$ symbols in a $\mathrm{RB}$ of the normal $\mathrm{CP}$.

If a user can take the highest $\mathrm{BW}$ of $3 \mathrm{RB}$ then we can represent the traffic by the state transition chain of Figure 6, where RB arrival rate is $\lambda$ calls/unit time and its termination rate is $\mu$. If $k$ is the highest allowed BW or RB then each probability state will emit $k$ arrivals and maximum possible terminate rate will 


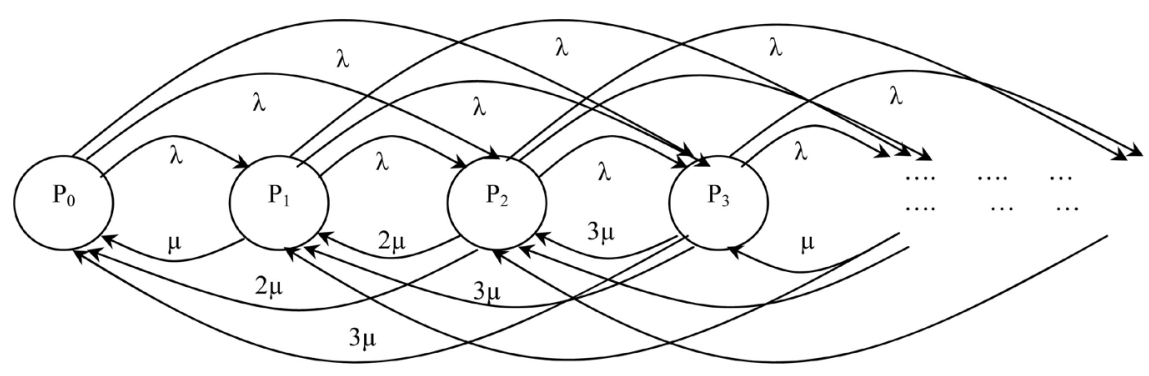

Figure 6. Markov chain of LTE user with variable BW.

be $k \mu$. Applying node equation on each node we get 9 linear equations. Using normalization of entire sample space, we get the following relations.

Applying node equation on nodes of Figure 6 we get,

$$
P_{x}(3 A+3 x)=(x+1) P_{x+1}+(x+2) P_{x+2}+(x+3) P_{x+3}+\left(P_{x-3}+P_{x-2}+P_{x-1}\right) A,
$$

for $3 \leq x \leq m-3, m$ is the last state of the chain.

$$
\begin{gathered}
P_{m-2}\{2 A+3(m-2)\}=(m-1) P_{m-1}+m P_{m}+\left(P_{m-5}+P_{m-4}+P_{m-3}\right) A ; x=m-2(28) \\
P_{m-1}(A+3(m-1))=m P_{m}+\left(P_{m-4}+P_{m-3}+P_{m-2}\right) A ; x=m-1 \\
m P_{m}=\left(P_{m-3}+P_{m-2}+P_{m-1}\right) A ; x=m
\end{gathered}
$$

For entire sampling space,

$$
\sum_{i=0}^{m} P_{i}=1
$$

If normalized probability state of the chain is $q_{x}$ then, the blocking probability of a user uses $k \mathrm{RB}$ will be,

$$
B(k)=\sum_{i=0}^{k} q_{m-i}
$$

Combination of analysis of three subsections: $3.1,3.2$ and 3.3 provide the exact traffic model of a LTE network.

\section{Results}

This section provides the analytical results based on the statistical model of Section 3 and verified by simulation. Taking the following traffic parameters: $\mu_{m}=$ $\mu_{f}=1.2$ calls $/ \mathrm{min}, v_{f}=10 \mathrm{~m} / \mathrm{s}, R_{f}=20 \mathrm{~m}, V_{m}=100 \mathrm{~m} / \mathrm{min}, R_{m}=1 \mathrm{Km}$ and $B_{f}=$ 0.02 , we obtain the probability of handover to femto and macrocells as: $P_{h f}=0.21$ and $P_{h m}=0.05$ respectively. Figure 7 shows the profile of probability of $F T$ (both the cases i.e. when a UE starts its session at macro and femto cell) against the blocking probability of macrocell taking the velocity of users at macro and femtocell as the parameters. The probability of $F T$ increases with increase in both $B_{m}$ and velocity of the users. Again taking, $\mu_{f}=1.8$ calls $/ \mathrm{min}, v=10 \mathrm{~m} / \mathrm{min}, R_{f}=20$ $\mathrm{m}$, we get $P_{h f}=0.15$. For another set of traffic parameters: $\mu_{f}=1.2 \mathrm{calls} / \mathrm{min}, V=$ $12.5 \mathrm{~m} / \mathrm{min}, R_{f}=20 \mathrm{~m}$, we get $P_{h f}=0.25$. Considering above two sets of parameters, the variation of $F T$ against arrival rate of femtocell is shown in Figure 8. 


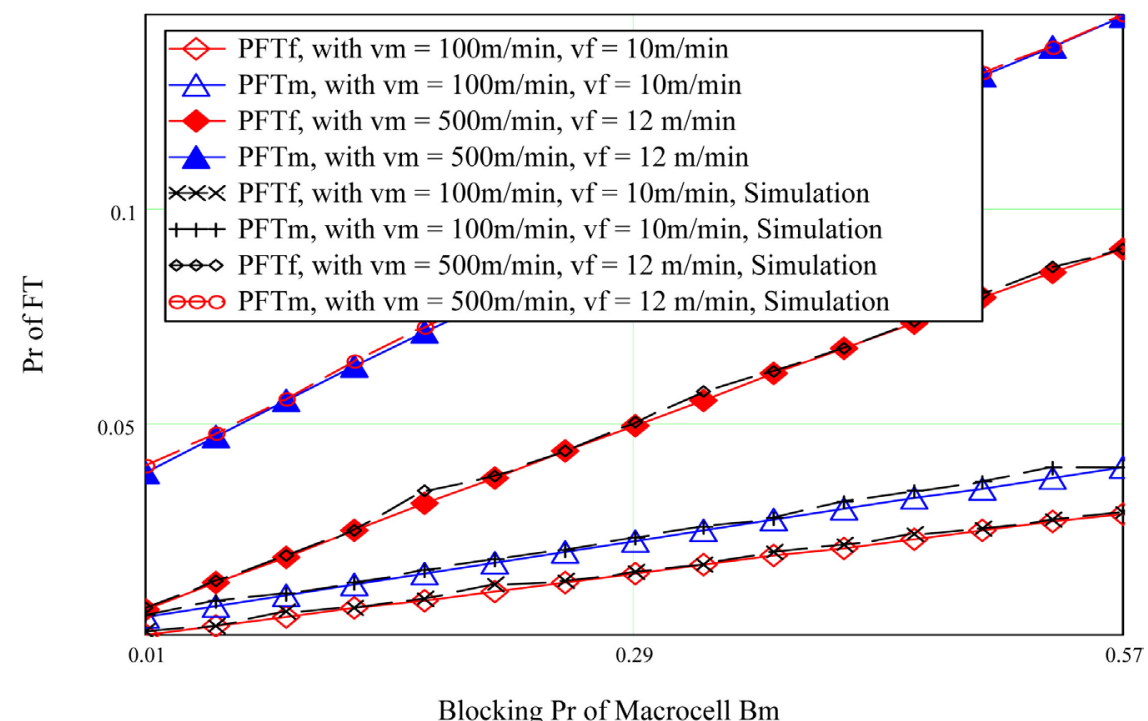

Figure 7. Variation of $\mathrm{P} F$ T against Bm.

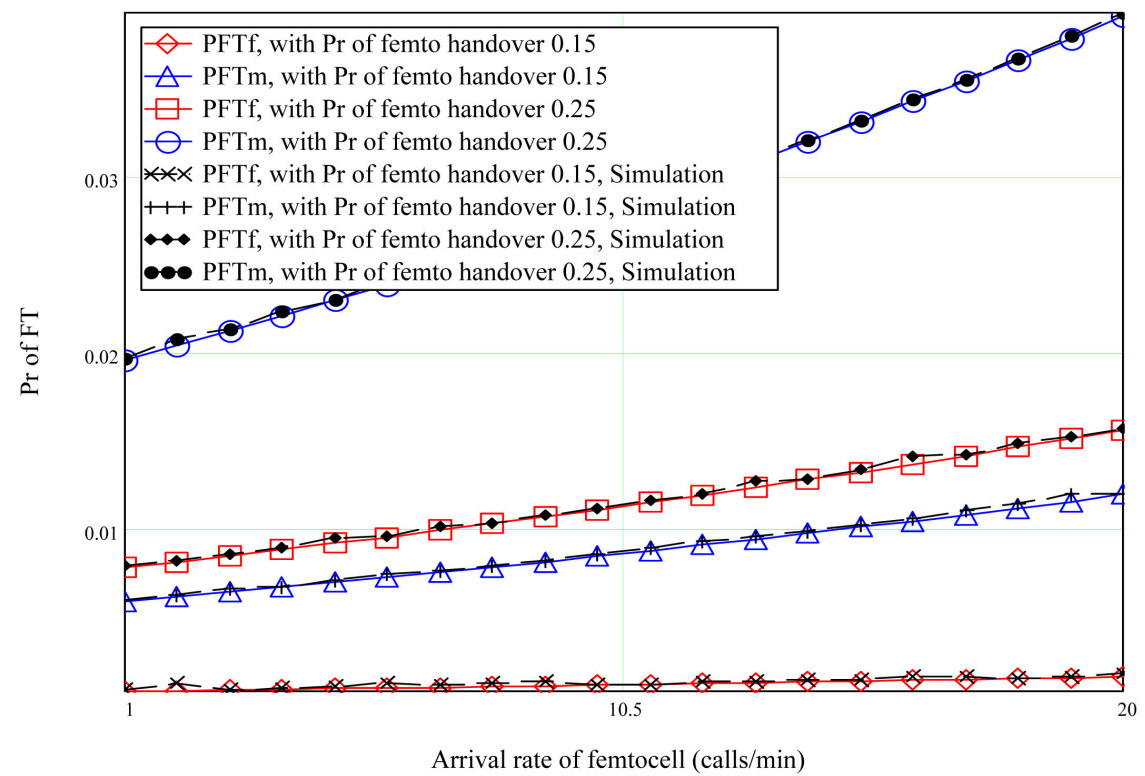

Figure 8. Variation of $\mathrm{P} F T$ against $\lambda_{f}$

Almost similar profile is found as Figure 7 i.e. the $P_{F T}$ increases with increase in both the $\lambda_{f}$ and $P_{h f}$

Next, the probability of $F T$ is varied against the number of channels of femtocell taking the same traffic parameters shown in Figure 9. In this case, the probability of $F T$ decreases with increase in the number of channels of femtocell. All the analytical results are verified by simulation which provides more than $95 \%$ confidence level.

The next part of the result section is related to subsection 3.2; where the traffic parameters are combined with fading parameters of MIMO channel to measure the performance of the network. The variation of $P_{F T}$ against the number of channels of femtocell is shown in Figure 10(a) for average SNR of $-2 \mathrm{~dB}$ and (b) 


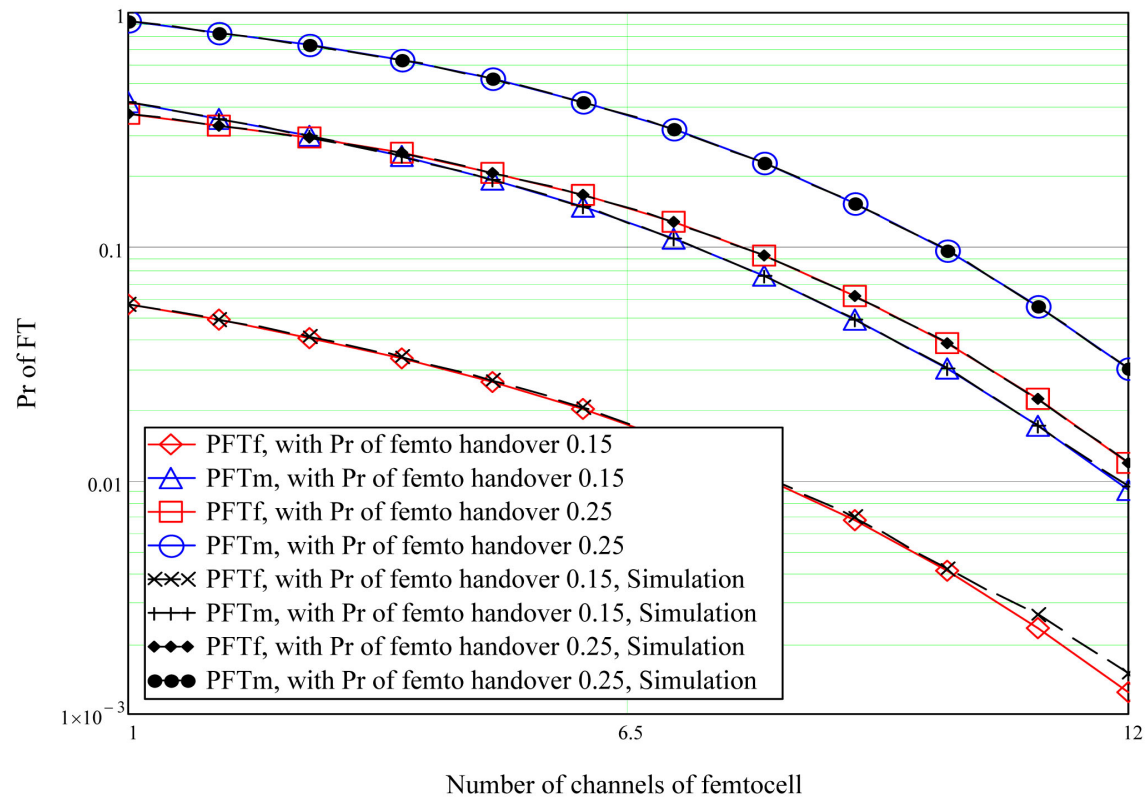

Figure 9. Variation of probability of FTagainst the number of channels of femtocell.

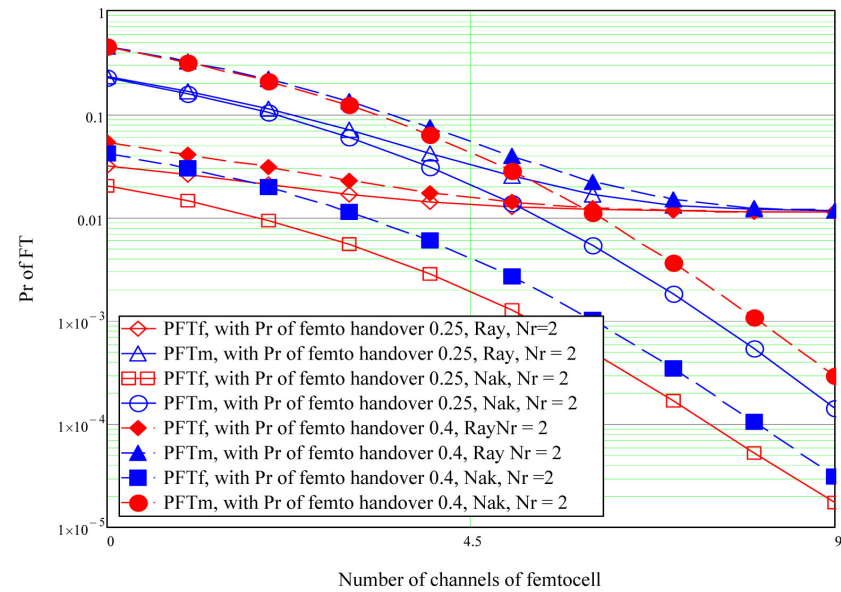

(a)

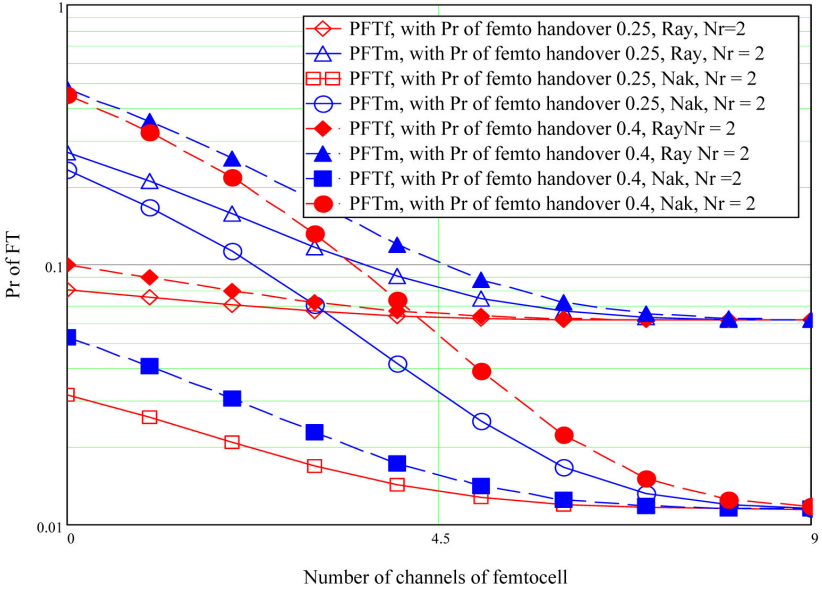

(b)

Figure 10. Variation of FTagainst the number of channels of femtocell.

for average SNR of $2 \mathrm{~dB}$ under STBC taking the probability of handover of femtocell as a parameter. The $P_{F T}$ decreases with increase in the number of channels of femtocell but increases with increase in the probability of handover of femtocells. The channel parameters are taken as: average $\mathrm{SNR}=-2 \mathrm{~dB}$ and $2 \mathrm{~dB}$, the standard deviation of channel gain $\sigma=0.83$, the parameters of Nakagami- $m$ fading $m=2, N_{r}=2$. The traffic parameters are taken as: number of femto cells $N_{f}=12, \lambda_{f}=9 \mathrm{calls} / \mathrm{min}, \lambda_{h f}=4 \mathrm{calls} / \mathrm{min}, \lambda_{m}=20 \mathrm{calls} / \mathrm{min}, \lambda_{h m}=12 \mathrm{calls} / \mathrm{min}$, $\mu_{m}=4.2$ calls $/ \mathrm{min}$ and $\mu_{f}=2$ calls $/ \mathrm{min}$. The performance is found better under Nakagami- $m$ fading case compared to the Rayleigh fading environment because of $m$ direct link (between transmitter and receiver) of Nakagami- $m$ fading. Under Rayleigh fading environment three is no direct link between transmitter and receiver hence performance is found very poor at low SNR but when the received 


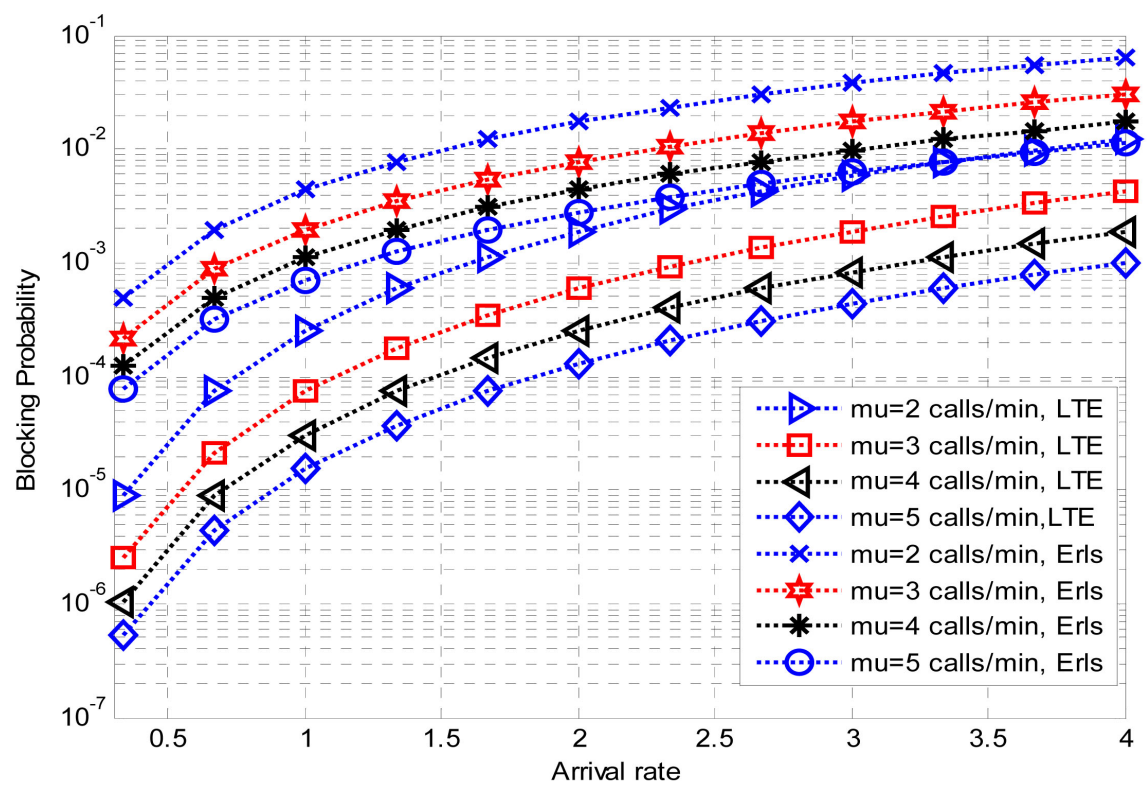

Figure 11. Comparison of proposed LTE traffic.

SNR attains above a threshold the performance of Rayleigh and Nakagami- $m$ environment are merged. The difference between performance at low $(-2 \mathrm{~dB})$ and high (2dB) SNR is found distinct in Figure 10(a) and Figure 10(b).

Finally, the call blocking probability of variable BW traffic of LTE and Erlang's model are compared in Figure 11. Here Blocking probability is varied against call arrival rate taking termination rate as a parameter provided the number of channels $n=8$. The blocking probability of variable BW traffic of LTE is found smaller than the case of Erlang's traffic. The arrival rate of the state transition chain of Figure 6 is $\lambda$ for any transition, hence each state experiences the total arrival of $3 \lambda$, on the other hand the termination rate from probability state $\mathrm{Px}$ is $3 \mathrm{x} \mu$. The termination rate of the proposed model is more prominent than the Erlang's model of $\mathrm{M} / \mathrm{M} / \mathrm{n} / \mathrm{K}$; hence blocking probability of the proposed model is found smaller than the conventional Erlag's model.

\section{Conclusion}

The paper combines the traffic model with the outage probability of wireless link under two different small scale fading cases. A new traffic model of variable BW (variable RB for up or down link) is proposed based on Markov chain and comparison is made with $\mathrm{M} / \mathrm{M} / \mathrm{n} / \mathrm{K}$ traffic. In future we will combine the wireless link parameters with our proposed traffic model of variable BW. In this paper we consider the pdf of SNR in determining the outage probability but we have the scope to use the pdf of SIR (signal to interference ratio) or SINR to evaluate the same parameter. In this case $S I N R$ will be a random variable as the ratio of another two random variables; hence the concept of mixed random variable can be applied for it. We consider $\mathrm{M} / \mathrm{M} / \mathrm{n} / \mathrm{K}$ traffic model throughout the paper but in voice data integrated traffic we can consider M/G/1/K traffic model as well. 


\section{Conflicts of Interest}

The authors declare no conflicts of interest regarding the publication of this paper.

\section{References}

[1] Pyattaev, A., Johnsson, K., Andreev, S. and Koucheryavy, Y. (2013) 3GPP LTE Traffic Offloading onto WiFi Direct. IEEE WCNC Workshop on Mobile Internet. Traffic Modeling, Subscriber Perception Analysis and Traffic Aware Network Design, Shanghai, 7-10 April 2013, 135-140. https://doi.org/10.1109/WCNCW.2013.6533328

[2] Hagos, D.H. (2015) The Performance of Network-Controlled Mobile Data Offloading from LTE to WiFi Networks. Springer Science + Business Media, New York, 675-694. https://doi.org/10.1007/s11235-015-0061-2

[3] Adesh, N.D. and Renuka, A. (2019) Adaptive Receiver-Window Adjustment for Delay Reduction in LTE Networks. Journal of Computer Networks and Communications, 2019, Article ID: 3645717. https://doi.org/10.1155/2019/3645717

[4] Joseph, J., Ruhl, R. and Samuel, H. (2018) Reducing Traffic Congestion for Machine to Machine Type Communication over 4G-LTE Network by Decreasing Total Bytes Transmitted. 17 th IEEE International Conference on Trust, Security and Privacy in Computing and Communications/12 th IEEE International Conference on Big Data Science and Engineering, New York, 1-3 August 2018, 1720-1721. https://doi.org/10.1109/TrustCom/BigDataSE.2018.00259

[5] Muntadher, A., Amir, E. and Nidal, N. (2017) Traffic Density Based Adaptive QoS Classes Mapping for Integrated LTE-WiMAX 5G Networks. IEEE ICC Mob and Wireless Net, Paris, 21-25 May 2017, 1-7.

[6] Kankare, M., Asp, A., Sydorov, Y., Niemelä, J. and Valkama, M. (2013) Large-Scale Femtocell Network Deployment and Measurements. 6th International Workshop on Multiple Access Communications, Lithuania, December 2013, 100-112. https://doi.org/10.1007/978-3-319-03871-1_10

[7] Bouras, C. and Diles, G. (2017) Distributed Sleep Mode Power Control in 5G Ultra Dense Networks. WWIC, St. Petersburg, 21-23 June 2017, 65-76. https://doi.org/10.1007/978-3-319-61382-6_6

[8] Nguyen, D.-H. and Nguyen, H. (2016) A Dynamic Rate Adaptation Algorithm Using WB E-Model for Voice Traffic over LTE Network. IEEE Conference on Wireless Days, Toulouse, 1-6 March 2016, 1-6. https://doi.org/10.1109/WD.2016.7461490

[9] Pawar, A.R., Bhardwaj, S.S. and Wandre, S.N. (2015) Mobile Data Offloading Techniques and Related Issues. International Journal of Advanced Research in Computer Engineering \& Technology, 4, 1367-1371.

[10] Ruiz, J.M., Matías, T. and Luna, S. (2015) A Femtocell Location Strategy for Improving Adaptive Traffic Sharing in Heterogeneous LTE Networks. EURASIP Journal on Wireless Communications and Networking, 2015, Article No. 38. https://doi.org/10.1186/s13638-015-0246-0

[11] Chowdhury, M.Z. and Jang, Y.M. (2013) Handover Management in High-Dense Femtocellular Networks. EURASIP Journal on Wireless Communications and Networking, 2013, Article No. 6. https://doi.org/10.1186/1687-1499-2013-6

[12] Markova, E., Moltchanov, D., Gudkova, I., Samouylov, K. and Koucharyavy, Y. (2019) Performance Assessment of QoS-Aware LTE Sessions Offloading onto LAA/WiFi 
Systems. IEEE Access, 7, 36300-36311. https://doi.org/10.1109/ACCESS.2019.2905035

[13] Islam, I., Rabbi, F., Khan, R.T. and Akhter, J. (2015) Performance Evaluation of Cognitive Radio Network Based on 2-D Markov Chain. Journal of Telecommunications and Information Technology, 3, 39-44.

[14] Chaturvedi, A. and Solanki, A. (2012) Analyzing Area Spectral Efficiency and Its Impacts on the Total Spectrum Requirements for IMT Advanced. International Journal of Distributed and Parallel Systems, 3, 141-152. https://doi.org/10.5121/ijdps.2012.3212

[15] Oh, E. (2015) Distributed Cell Configuration Strategy for Macro/Micro Overlaid Hierarchical Cellular Systems. International Journal of Software Engineering and Its Applications, 9, 177-188. https://doi.org/10.14257/ijseia.2015.9.9.15

[16] Islam, I., Das, J.K. and Hossain, S. (2007) Modeling of Voice Data Integrated Traffic in $3 \mathrm{G}$ Mobile Cellular Network. Journal of Telecommunications and Information Technology, 2, 103-108.

[17] Jun, K. and Kim, S. (2014) Modeling and Performance Analysis for Cell Access and Handoff Schemes in Two-Tier Cellular Networks. Mathematical Problems in Engineering, 2014, Article ID: 860729. https://doi.org/10.1155/2014/860729

[18] Hossain, M.A., Islam, M.I. and Amin, M.R. (2016) Load Sensitive Power Saving Technique for 4G Mobile Network under Limited User Traffic. Communications and Network, 8, 79-87. https://doi.org/10.4236/cn.2016.82009

[19] Jabbari, B. (1996) Teletraffic Aspect of Evolving and Next Generation Wireless Communication Networks. IEEE Personal Communications, 3, 4-9. https://doi.org/10.1109/98.556473

[20] Ortigoza-Guerrero, L. and Hamid Aghvami, A. (1999) A Prioritized Handoff Dynamic Channel Allocation Strategy for PCS. IEEE Transaction on Vehicular Technology, 48, 1203-1215. https://doi.org/10.1109/25.775369

[21] Matthaiou, M. and Zhong, C. (2012) Low-SNR Analysis of MIMO Weibull Fading Channels. IEEE Communications Letters, 16, 694-697. https://doi.org/10.1109/LCOMM.2012.030912.120227

[22] Hashem, T. and Islam, M.I. (2014) Performance Analysis of MIMO Link under Fading Channels. 17 th International Conference on Computer and Information Technology, Dhaka, December 2014, 498-503. https://doi.org/10.1109/ICCITechn.2014.7073080

[23] Da Costa, D.B., Ferdinand, N.S., Dias, U.S., de Sousa Jr., R.T. and Latva-aho, M. (2016) Secrecy Outage Performance of MIMO Wiretap Channels with Multiple Jamming Signals. Journal of Communications and Information Systems, 31, 30-40. https://doi.org/10.14209/jcis.2016.2

[24] Li, Y.Z., Zhang, L., Cimini Jr., L.J. and Zhang, H.L. (2011) Statistical Analysis of MIMO Beamforming with Co-Channel Unequal-Power MIMO Interferers under Path-Loss and Rayleigh Fading. IEEE Transactions on Signal Processing, 59, 3738-3748. https://doi.org/10.1109/TSP.2011.2155654

[25] Hung, C.-C., Chiang, C.-T., Wu, R.-C. and Lee, C. (2012) Positive Effect of Severe Nakagami- $m$ Fading on the Performance of Multiuser TAS/MRC Systems with High Selection Gain. International Journal of Antennas and Propagation, 2012, Article ID: 987631. 